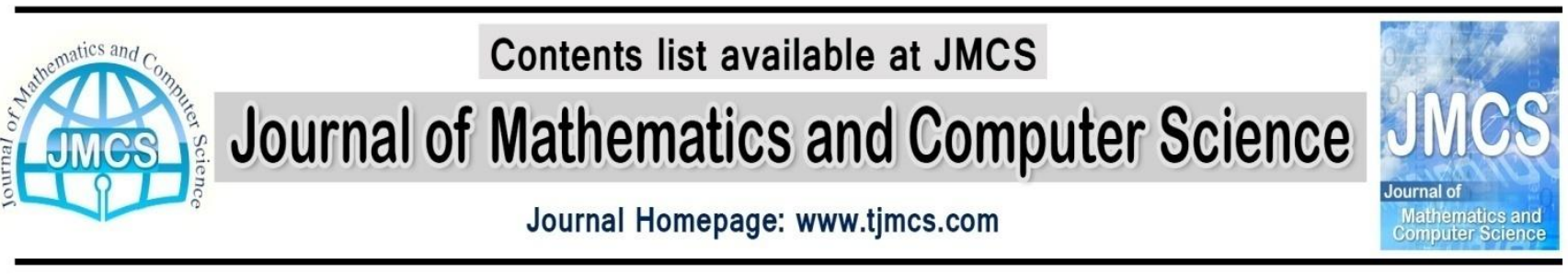

\title{
Non-Archimedean Intuitionistic Fuzzy Continuity of
}

\section{Dectic Mappings}

\author{
Mohammad Ali Abolfathi ${ }^{1}$, Ali Ebadian ${ }^{2}$, Rasoul Aghalary ${ }^{3}$ \\ ${ }^{1,2,3}$ Department of Mathematics, Faculty of Science, Urmia University, P.O. BOX 165, Urmia, Iran \\ E-mail: $\underline{\text { m.abolfathi@urmia.ac.ir }}^{1}$; E-mail: ebadian.ali@gmail.com ${ }^{2}$; \\ E-mail: r.aghalary@urmia.ac.ir ${ }^{3}$
}

Article history:

Received March 2014

Accepted March 2014

Available online April 2014

\begin{abstract}
In this paper, we investigate the non-Archimedean intuitionistic fuzzy continuity through the existence of a certain solution of a fuzzy stability problem for the system of additive-quadratic-cubic-quartic functional equations.
\end{abstract}

Keywords: Non-Archimedean intuitionistic fuzzy normed spaces, Mixed type functional equation, Dectic mappings, HyersUlam stability.

\section{Introduction}

The study of stability problems for functional equations is related to a question of Ulam [25] concerning the stability of group homomorphisms and affirmatively answered for Banach spaces by Hyers [12]. The results of Hyers were generalized by Aoki [2], Rassias [21] and Gavruta [10], respectively. We refer the reader to see, for instance, $[1,4,5,6,7,8,9,24]$ for more information on different aspects of stability of functional equations. The notion of intuitionistic fuzzy set introduced by Atanassov [3] has been used extensively in many areas of mathematics and sciences. The notion of intuitionistic fuzzy norm $[22,13,20]$ is also useful one to deal with the inexactness and vagueness arising in modeling. There are many situations where the norm of a vector is not possible to find and the concept of intuitionistic fuzzy norm seems to be more suitable in such cases, that is, we can deal with such situations by modeling the inexactness through the intuitionistic fuzzy norm. The stability problem for Pexiderized quadratic functional equation, Jensen functional equation, cubic functional equation, functional equations associated with inner product spaces, and additive functional equation was considered in $[14,15,16,17,18,23]$, respectively, in the intuitionistic fuzzy normed spaces. 
In this paper, we determine some stability results concerning the system of additive-quadratic-cubicquartic functional equations

$$
\left\{\begin{array}{l}
f\left(a x_{1}+b x_{2}, y, z, w\right)+f\left(a x_{1}-b x_{2}, y, z, w\right)=2 a f\left(x_{1}, y, z, w\right) \\
f\left(x, a y_{1}+b y_{2}, z, w\right)+f\left(x, a y_{1}-b y_{2}, z, w\right)=2 a^{2} f\left(x, y_{1}, z, w\right)+2 b^{2} f\left(x, y_{2}, z, w\right) \\
f\left(x, y, a z_{1}+b z_{2}, w\right)+f\left(x, y, a z_{1}-b z_{2}, w\right)=a b^{2}\left(f\left(x, y, z_{1}+z_{2}, w\right)\right. \\
\left.\quad+f\left(x, y, z_{1}-z_{2}, w\right)\right)+2 a\left(a^{2}-b^{2}\right) f\left(x, y, z_{1}, w\right) \\
\quad f\left(x, y, z, a w_{1}+b w_{2}\right)+f\left(x, y, z, a w_{1}-b w_{2}\right)=a^{2} b^{2}\left(f\left(x, y, z, w_{1}+w_{2}\right)\right. \\
\left.\quad+f\left(x, y, z, w_{1}-w_{2}\right)\right)+2 a^{2}\left(a^{2}-b^{2}\right) f\left(x, y, z, w_{1}\right)-2 b^{2}\left(a^{2}-b^{2}\right) f\left(x, y, z, w_{2}\right)
\end{array}\right.
$$

where $a, b \in \mathbb{Z} \backslash\{0\}$ with $a \neq \pm 1, \pm b$, in the setting of non-Archimedean intuitionistic fuzzy normed spaces. We also study the non-Archimedean intuitionistic fuzzy continuity through the existence of a certain solution of a fuzzy stability problem for the system of additive-quadratic-cubic-quartic functional equations. Note that, the function $f: \mathbb{R} \times \mathbb{R} \times \mathbb{R} \times \mathbb{R} \rightarrow \mathbb{R}$ given by $f(x, y, z, w)=c x y^{2} z^{3} w^{4}$ is a solution of (1.1). In particular, putting $x=y=z=w$, we get a dectic function $g: \mathbb{R} \rightarrow \mathbb{R}$ in one variable given by $g(x):=f(x, x, x, x)=c x^{10}$. The proof of the following proposition is evident, and we omit the details.

Proposition 1.1. Let $X$ and $Y$ be real linear spaces. If a mapping $f: X \times X \times X \times X \rightarrow Y$ satisfies system (1.1), then $f(\lambda x, \mu y, \eta z, \gamma w)=\lambda \mu^{2} \eta^{3} \gamma^{4} f(x, y, z, w)$ for all $\mathrm{x}, \mathrm{y}, \mathrm{z}, \mathrm{w} \in X$, and all rational numbers $\lambda, \mu, \eta, \gamma$.

\section{Non-Archimedean intuitionistic fuzzy normed spaces}

In this section, we introduce the concept of non-Archimedean intuitionistic fuzzy normed space and further define the notions of convergence and Cauchy sequences in this new framework.

A valuation is a map $|$.$| from a field K$ into $[0, \infty)$ such that 0 is the unique element having the 0 valuation, $\left|k_{1} k_{2}\right|=\left|k_{1}\right|\left|k_{2}\right|$, and the triangle inequality holds, that is, $\left|k_{1}+k_{2}\right| \leq\left|k_{1}\right|+\left|k_{2}\right|$, for all $k_{1}, k_{2} \in \mathrm{K}$. We say that a field $\mathrm{K}$ is valued if $\mathrm{K}$ carries a valuation. The usual absolute values of $\mathbb{R}$ and $\mathbb{C}$ are examples of valuations.

Let us consider a valuation which satisfies stronger condition than the triangle inequality. If the triangle inequality is replaced by $\left|k_{1}+k_{2}\right| \leq \max \left\{\left|k_{1}\right|,\left|k_{2}\right|\right\}$ for all $k_{1}, k_{2} \in \mathrm{K}$ then, a map $|$.$| is$ called non-Archimedean or ultrametric valuation, and field is called a non-Archimedean field. Clearly $|1|=|-1|=1$ and $|n| \leq 1$, for all $n \in \mathbb{N}$. A trivial example of a non-Archimedean valuation is the map $|$.$| taking everything but 0$ into 1 and $|0|=0$.

Let $X$ be a vector space over a field $\mathrm{K}$ with a non-Archimedean valuation |.|. A non-Archimedean normed space is a pair $(X,\|\cdot\|)$, where $\|\cdot\|: X \rightarrow[0, \infty)$ is such that

(i) $\|\cdot\|=0$, if and only if $x=0$;

(ii) $\|\lambda x\|=\lambda\|x\|$, for all $\lambda \in \mathrm{K}$ and

(iii) the strong triangle inequality, $\left\|k_{1}+k_{2}\right\| \leq \max \left\{\left\|k_{1}\right\|,\left\|k_{2}\right\|\right\}$ for all $x, y \in X$.

In [11], Hensel discovered the $p$-adic numbers as a number theoretical analogue of power series in complex analysis. The most interesting example of non-Archimedean spaces is $p$-adic numbers. 
A binary operation $*:[0,1] \times[0,1] \rightarrow[0,1]$ is said to be a continuous $t$-norm if it satisfies the following conditions:

(a) $*$ is associative and commutative, (b) $*$ is continuous, (c) $a * 1=a$ for all $a \in[0,1]$ and (d) $a * b \leq$ $c * d$ whenever $a \leq c$ and $b \leq d$ for each $a, b, c, d \in[0,1]$.

A binary operation $\diamond:[0,1] \times[0,1] \rightarrow[0,1]$ is said to be a continuous $t$-conorm if it satisfies the following conditions:

(a') $\diamond$ is associative and commutative, (b') $\diamond$ is continuous, (c') $a \diamond 0=a$ for all $a \in[0,1]$ and

(d') $a \diamond b \leq c \diamond d$ whenever $a \leq c$ and $b \leq d$ for each $a, b, c, d \in[0,1]$.

Definition 2.1. The five-tuple $(X, \mathcal{F}, \mathcal{P}, *, \diamond)$ is said to be a non-Archimedean intuitionistic fuzzy normed space (for short, non-Archimedean IFN space) if $X$ is a vector space over a non-Archimedean field $\mathrm{K}, *$ is a continuous $t$-norm, $\diamond$ is a continuous $t$-conorm, and $\mathcal{F}, \mathcal{P}$ are functions from $X \times \mathbb{R}$ to $[0,1]$ satisfying the following conditions. For every $x, y \in X$ and $s, t \in \mathrm{K}$,

(i) $\mathcal{F}(x, t)+\mathcal{P}(x, t) \leq 1$, (ii) $\mathcal{F}(x, t)>0$, (iii) $\mathcal{F}(x, t)=1$ if and only if $x=0$, (iv) $\mathcal{F}(\alpha x, t)=$ $\mathcal{F}\left(x, \frac{t}{|\alpha|}\right)$ for each $\alpha \neq 0$, (v) $\mathcal{F}(x, t) * \mathcal{F}(y, s) \leq \mathcal{F}(x+y$, $\max \{t, s\})$, (vi) $\mathcal{F}(x,):.(0, \infty) \rightarrow[0,1]$ is continuous, (vii) $\lim _{t \rightarrow \infty} \mathcal{F}(x, t)=1$ and $\lim _{t \rightarrow 0} \mathcal{F}(x, t)=0$, (viii) $\mathcal{P}(x, t)<1$, (ix) $\mathcal{P}(x, t)=0$ if and only if $x=0$, (x) $\mathcal{P}(\alpha x, t)=\mathcal{P}\left(x, \frac{t}{|\alpha|}\right)$ for each $\alpha \neq 0$, (xi) $\mathcal{P}(x, t) \diamond \mathcal{P}(y, s) \geq P(x+y$, max $\{t, s\}$ ), (xii) $\mathcal{P}(x,):.(0, \infty) \rightarrow[0,1]$ is continuous, (xiii) $\lim _{t \rightarrow \infty} \mathcal{P}(x, t)=0$ and $\lim _{t \rightarrow 0} \mathcal{P}(x, t)=1$.

In this case $(\mathcal{F}, \mathcal{P})$ is called a non-Archimedean intuitionistic fuzzy norm.

Example 2.2. Let $(X,\|\|$.$) be a non-Archimedean normed space, a * b=a b$ and $a \diamond b=$ $\min \{a+b, 1\}$ for all $a, b \in[0,1]$. For all $x \in X$, all $t>0$ and $k=1,2$, consider the following:

$\mathcal{F}_{k}(x, t)=\left\{\begin{array}{cc}\frac{t}{t+k\|x\|} & t>0 \\ 0 & t \leq 0,\end{array} \quad\right.$ and $\quad \mathcal{P}_{k}(x, t)=\left\{\begin{array}{cc}\frac{k\|x\|}{t+k\|x\|} & t>0 \\ 0 & t \leq 0 .\end{array}\right.$

Then $\left(X, \mathcal{F}_{k}, \mathcal{P}_{k}, *, \diamond\right)$ is a non-Archimedean intuitionistic fuzzy normed space.

Definition 2.3. Let $(X, \mathcal{F}, \mathcal{P}, *, \diamond)$ be a non-Archimedean intuitionistic fuzzy normed space. Then, a sequence $s=\left(s_{n}\right)$ is said to be

(i) convergent in $(X, \mathcal{F}, \mathcal{P}, *, \diamond)$ or simply $(\mathcal{F}, \mathcal{P})$-convergent to $\lambda \in X$ if for every $\epsilon>0$ and $t>0$, there exists $n_{0} \in \mathbb{N}$ such that $\mathcal{F}\left(s_{n}-\lambda, t\right)>1-\epsilon$ and $\mathcal{P}\left(s_{n}-\lambda, t\right)<\epsilon$ for all $n \geq n_{0}$. In this case we write $(\mathcal{F}, \mathcal{P})-\lim _{n \rightarrow \infty} s_{n}=\lambda$ and $\lambda$ is called the $(\mathcal{F}, \mathcal{P})$ - limit of $s=\left(s_{n}\right)$.

(ii) Cauchy in $(X, \mathcal{F}, \mathcal{P}, *, \diamond)$ or simply $(\mathcal{F}, \mathcal{P})$-Cauchy if for every $\epsilon>0$ and $t>0$, there exists $n_{0} \in \mathbb{N}$ such that $\mathcal{F}\left(s_{n}-s_{m}, t\right)>1-\epsilon$ and $\mathcal{P}\left(s_{n}-s_{m}, t\right)<\epsilon$ for all $n \geq n_{0}$. A non-Archimedean IFN-space $(X, \mathcal{F}, \mathcal{P}, *, \diamond)$ is said to be complete if every $(\mathcal{F}, \mathcal{P})$ - Cauchy is $(\mathcal{F}, \mathcal{P})$ - convergent. In this case $(X, \mathcal{F}, \mathcal{P}, *, \diamond)$ is called non-Archimedean intuitionistic fuzzy Banach space.

Throughout this paper, we assume that $I=\{1,2,3,4\}$.

\section{Non-Archimedean intuitionistic fuzzy stability}

In this section, we deal with the stability results concerning for system of functional equations (1.1) in non-Archimedean intuitionistic fuzzy normed spaces. 
Theorem 3.1. Let $X$ be a linear space over a non-Archimedean field $K$ and let $\left(Z, \mathcal{F}^{\prime}, \mathcal{P}^{\prime}\right)$ be a nonArchimedean IFN space. Suppose that $\varphi_{i}: X \times X \times X \times X \times X \rightarrow Z$ for $i \in$ be functions such that $|a|<1$ is far from zero and for some real number $\alpha$ with $|\alpha|>|a|^{10}$,

$$
\left\{\begin{array}{l}
\mathcal{F}^{\prime}\left(\varphi_{i}\left(\frac{x}{a}, \frac{y}{a}, \frac{z}{a}, \frac{w}{a}, \frac{v}{a}, \mathrm{t}\right) \geq \mathcal{F}^{\prime}\left(\varphi_{i}(x, y, z, w, v),|\alpha| \mathrm{t}\right)\right. \\
\mathcal{P}^{\prime}\left(\varphi_{i}\left(\frac{x}{a}, \frac{y}{a}, \frac{z}{a}, \frac{w}{a}, \frac{v}{a}, \mathrm{t}\right) \leq \mathcal{P}^{\prime}\left(\varphi_{i}(x, y, z, w, v),|\alpha| \mathrm{t}\right)\right.
\end{array}\right.
$$

for all $x, y, z, w, v \in X, t>0$ and $i \in I$. Let $f$ be a mapping from $X \times X \times X \times X$ to a nonArchimedean intuitionistic fuzzy Banach space $(Y, \mathcal{F}, \mathcal{P})$ such that $f(x, 0, z, w)=0, f(x, y, z, 0)=0$ for all $x, y, z, w \in X$, and satisfying

$$
\left\{\begin{array}{l}
\mathcal{F}\left(f\left(a x_{1}+b x_{2}, y, z, w\right)+f\left(a x_{1}-b x_{2}, y, z, w\right)-2 a f\left(x_{1}, y, z, w\right), t\right) \\
\geq \mathcal{F}^{\prime}\left(\varphi_{1}\left(x_{1}, x_{2}, y, z, w\right), t\right) \\
\mathcal{P}\left(f\left(a x_{1}+b x_{2}, y, z, w\right)+f\left(a x_{1}-b x_{2}, y, z, w\right)-2 a f\left(x_{1}, y, z, w\right), t\right) \\
\leq \mathcal{P}^{\prime}\left(\varphi_{1}\left(x_{1}, x_{2}, y, z, w\right), t\right)
\end{array}\right.
$$

$$
\left\{\begin{array}{l}
\mathcal{F}\left(f\left(x, a y_{1}+b y_{2}, z, w\right)+f\left(x, a y_{1}-b y_{2}, z, w\right)-2 a^{2} f\left(x, y_{1}, z, w\right)-2 b^{2} f\left(x, y_{2}, z, w\right), t\right) \\
\quad \geq \mathcal{F}^{\prime}\left(\varphi_{2}\left(x, y_{1}, y_{2}, z, w\right), t\right), \\
\mathcal{P}\left(f\left(x, a y_{1}+b y_{2}, z, w\right)+f\left(x, a y_{1}-b y_{2}, z, w\right)-2 a^{2} f\left(x, y_{1}, z, w\right)-2 b^{2} f\left(x, y_{2}, z, w\right), t\right) \\
\leq \mathcal{P}^{\prime}\left(\varphi_{2}\left(x, y_{1}, y_{2}, z, w\right), t\right),
\end{array}\right.
$$

$$
\begin{aligned}
& \left\{\begin{array}{l}
\mathcal{F}\left(f\left(x, y, a z_{1}+b z_{2}, w\right)+f\left(x, y, a z_{1}-b z_{2}, w\right)-a b^{2}\left(f\left(x, y, z_{1}+z_{2}, w\right)+f\left(x, y, z_{1}-z_{2}, w\right)\right)\right. \\
\left.\quad-2 a\left(a^{2}-b^{2}\right) f\left(x, y, z_{1}, w\right), t\right) \geq \mathcal{F}^{\prime}\left(\varphi_{3}\left(x, y, z_{1}, z_{2}, w\right), t\right), \\
\mathcal{P}\left(f\left(x, y, a z_{1}+b z_{2}, w\right)+f\left(x, y, a z_{1}-b z_{2}, w\right)-a b^{2}\left(f\left(x, y, z_{1}+z_{2}, w\right)+f\left(x, y, z_{1}-z_{2}, w\right)\right)\right. \\
\left.\quad-2 a\left(a^{2}-b^{2}\right) f\left(x, y, z_{1}, w\right), t\right) \leq \mathcal{P}^{\prime}\left(\varphi_{3}\left(x, y, z_{1}, z_{2}, w\right), t\right),
\end{array}\right. \\
& \text { and }
\end{aligned}
$$

$$
\left\{\begin{array}{l}
\mathcal{F}\left(f\left(x, y, z, a w_{1}+b w_{2}\right)+f\left(x, y, z, a w_{1}-b w_{2}\right)-a^{2} b^{2}\left(f\left(x, y, z, w_{1}+w_{2}\right)+f\left(x, y, z, w_{1}-w_{2}\right)\right)\right. \\
\left.-2 a^{2}\left(a^{2}-b^{2}\right) f\left(x, y, z, w_{1}\right)+2 b^{2}\left(a^{2}-b^{2}\right) f\left(x, y, z, w_{2}\right), t\right) \geq \mathcal{F}^{\prime}\left(\varphi_{4}\left(x, y, z, w_{1}, w_{2}\right), t\right), \\
\mathcal{P}\left(f\left(x, y, z, a w_{1}+b w_{2}\right)+f\left(x, y, z, a w_{1}-b w_{2}\right)-a^{2} b^{2}\left(f\left(x, y, z, w_{1}+w_{2}\right)+f\left(x, y, z, w_{1}-w_{2}\right)\right)\right. \\
\left.\quad-2 a^{2}\left(a^{2}-b^{2}\right) f\left(x, y, z, w_{1}\right)+2 b^{2}\left(a^{2}-b^{2}\right) f\left(x, y, z, w_{2}\right), t\right) \leq \mathcal{P}^{\prime}\left(\varphi_{4}\left(x, y, z, w_{1}, w_{2}\right), t\right),
\end{array}\right.
$$

for all $x, y, z, w, x_{1}, x_{2}, y_{1}, y_{2}, z_{1}, z_{2}, w_{1}, w_{2} \in X$ and all $t>0$. Then there exists a unique dectic mapping $D: X \times X \times X \times X \rightarrow Y$ such that

$$
\left\{\begin{array}{l}
\mathcal{F}(f(x, y, z, w)-D(x, y, z, w), t) \geq M\left(x, y, z, w,\left(|\alpha|-|a|^{10}\right) t\right) \\
\mathcal{P}(f(x, y, z, w)-D(x, y, z, w), t) \leq N\left(x, y, z, w,\left(|\alpha|-|a|^{10}\right) t\right)
\end{array}\right.
$$

for all $x, y, z, w \in X$ and $t>0$, where 


$$
\left\{\begin{aligned}
M(x, y, z, w, t) & =\mathcal{F}^{\prime}\left(\varphi_{1}(x, 0, y, z, w),|2||a|^{-9} t\right) * \mathcal{F}^{\prime}\left(\varphi_{2}(a x, y, 0, z, w),|2||a|^{-7} t\right) \\
& * \mathcal{F}^{\prime}\left(\varphi_{3} \quad(a x, a y, z, 0, w),|2||a|^{-4} t\right) * \mathcal{F}^{\prime}\left(\varphi_{4}(a x, a y, a z, w, 0),|2| t\right) \\
N(x, y, z, w, t) & =\mathcal{P}^{\prime}\left(\varphi_{1}(x, 0, y, z, w),|2||a|^{-9} t\right) \diamond \mathcal{P}^{\prime}\left(\varphi_{2}(a x, y, 0, z, w),|2||a|^{-7} t\right) \\
& \diamond \mathcal{P}^{\prime}\left(\varphi_{3}(a x, a y, z, 0, w),|2||a|^{-4} t\right) \diamond \mathcal{P}^{\prime}\left(\varphi_{4}(a x, a y, a z, w, 0),|2| t\right) .
\end{aligned}\right.
$$

Proof. Put $x_{2}=0$ and replace $x_{1}, y, z, w$ by $2 x, 2 y, 2 z, 2 w$ respectively, in (3.2) we get

$$
\left\{\begin{array}{l}
\mathcal{F}(f(2 a x, 2 y, 2 z, 2 w)-a f(2 x, 2 y, 2 z, 2 w), t) \geq \mathcal{F}^{\prime}\left(\varphi_{1}(2 x, 0,2 y, 2 z, 2 w),|2| t\right) \\
\mathcal{P}(f(2 a x, 2 y, 2 z, 2 w)-a f(2 x, 2 y, 2 z, 2 w), t) \leq \mathcal{P}^{\prime}\left(\varphi_{1}(2 x, 0,2 y, 2 z, 2 w),|2| t\right),
\end{array}\right.
$$

for all $x, y, z, w \in X$ and $t>0$. Put $y_{2}=0$ and replace $x, y_{1}, z, w$ by $2 a x, 2 y, 2 z, 2 w$ respectively, in (3.3) we get

$\left\{\begin{array}{l}\mathcal{F}\left(f(2 a x, 2 a y, 2 z, 2 w)-a^{2} f(2 a x, 2 y, 2 z, 2 w), t\right) \geq \mathcal{F}^{\prime}\left(\varphi_{2}(2 a x, 2 y, 0,2 z, 2 w),|2| t\right) \\ \mathcal{P}\left(f(2 a x, 2 a y, 2 z, 2 w)-a^{2} f(2 a x, 2 y, 2 z, 2 w), t\right) \leq \mathcal{P}^{\prime}\left(\varphi_{2}(2 a x, 2 y, 0,2 z, 2 w),|2| t\right),\end{array}\right.$

for all $x, y, z, w \in X$ and $t>0$. Put $z_{2}=0$ and replace $x, y, z_{1}, w$ by $2 a x, 2 a y, 2 z, 2 w$ respectively, in (3.4) we get

$\left\{\begin{array}{l}\mathcal{F}\left(f(2 a x, 2 a y, 2 a z, 2 w)-a^{3} f(2 a x, 2 a y, 2 z, 2 w), t\right) \geq \mathcal{F}^{\prime}\left(\varphi_{3}(2 a x, 2 a y, 2 z, 0,2 w),|2| t\right) \\ \mathcal{P}\left(f(2 a x, 2 a y, 2 a z, 2 w)-a^{3} f(2 a x, 2 a y, 2 z, 2 w), t\right) \leq \mathcal{P}^{\prime}\left(\varphi_{3}(2 a 2 a x, 2 a y, 2 z, 0,2 w),|2| t\right),\end{array}\right.$

for all $x, y, z, w \in X$ and $t>0$. Put $w_{2}=0$ and replace $x, y, z, w_{1}$ by $2 a x, 2 a y, 2 a z, 2 w$ respectively, in (3.5) we get

$\left\{\begin{array}{l}\mathcal{F}\left(f(2 a x, 2 a y, 2 a z, 2 a w)-a^{4} f(2 a x, 2 a y, 2 a z, 2 w), t\right) \geq \mathcal{F}^{\prime}\left(\varphi_{4}(2 a x, 2 a y, 2 a z, 2 w, 0),|2| t\right) \\ \mathcal{P}\left(f(2 a x, 2 a y, 2 a z, 2 a w)-a^{4} f(2 a x, 2 a y, 2 a z, 2 w), t\right) \leq \mathcal{P}^{\prime}\left(\varphi_{4}(2 a x, 2 a y, 2 a z, 2 w, 0),|2| t\right)\end{array}\right.$

for all $x, y, z, w \in X$ and $t>0$. Thus for all $x, y, z, w \in X$ and $t>0$, we have

$$
\left\{\begin{aligned}
\mathcal{F}\left(f(2 a x, 2 a y, 2 a z, 2 a w)-a^{10} f(2 x, 2 y, 2 z, 2 w), t\right) \\
\quad \geq \mathcal{F}^{\prime}\left(\varphi_{1}(2 x, 0,2 y, 2 z, 2 w),|2||a|^{-9} t\right) * \mathcal{F}^{\prime}\left(\varphi_{2}(2 a x, 2 y, 0,2 z, 2 w),|2||a|^{-7} t\right) \\
\quad * \mathcal{F}^{\prime}\left(\varphi_{3}(2 a x, 2 a y, 2 z, 0,2 w),|2||a|^{-4} t\right) * \mathcal{F}^{\prime}\left(\varphi_{4}(2 a x, 2 a y, 2 a z, 2 w, 0),|2| t\right) \\
\mathcal{P}\left(f(2 a x, 2 a y, 2 a z, 2 a w)-a^{10} f(2 x, 2 y, 2 z, 2 w), t\right) \\
\quad \geq \mathcal{P}^{\prime}\left(\varphi_{1}(2 x, 0,2 y, 2 z, 2 w),|2||a|^{-9} t\right) \diamond \mathcal{P}^{\prime}\left(\varphi_{2}(2 a x, 2 y, 0,2 z, 2 w),|2||a|^{-7} t\right) \\
\quad \diamond \mathcal{P}^{\prime}\left(\varphi_{3}(2 a x, 2 a y, 2 z, 0,2 w),|2||a|^{-4} t\right) \diamond \mathcal{P}^{\prime}\left(\varphi_{4}(2 a x, 2 a y, 2 a z, 2 w, 0),|2| t\right) .
\end{aligned}\right.
$$

Replace $x, y, z, w$ by $\frac{x}{2}, \frac{y}{2}, \frac{z}{2}, \frac{w}{2}$ respectively, in (3.12) and using (3.1) we obtain 


$$
\left\{\begin{array}{l}
\mathcal{F}\left(f(a x, a y, a z, a w)-a^{10} f(x, y, z, w), t\right) \geq M(x, y, z, w, t) \\
\mathcal{P}\left(f(a x, a y, a z, a w)-a^{10} f(x, y, z, w), t\right) \leq N(x, y, z, w, t)
\end{array}\right.
$$

for all $x, y, z, w \in X$ and $t>0$. Replace $x, y, z, w$ by $\frac{x}{a^{k+1}}, \frac{y}{a^{k+1}}, \frac{z}{a^{k+1}}, \frac{w}{a^{k+1}}$ respectively, in (3.12) and using (3.1) we obtain

$$
\left\{\begin{array}{l}
\mathcal{F}\left(a^{10(k+1)} f\left(\frac{x}{a^{k+1}}, \frac{y}{a^{k+1}}, \frac{z}{a^{k+1}}, \frac{w}{a^{k+1}}\right)-a^{10 k} f\left(\frac{x}{a^{k}}, \frac{y}{a^{k}}, \frac{z}{a^{k}}, \frac{w}{a^{k}}\right),|a|^{10 k} t\right) \geq M\left(x, y, z, w,|\alpha|^{k+1} t\right) \\
\mathcal{P}\left(a^{10(k+1)} f\left(\frac{x}{a^{k+1}}, \frac{y}{a^{k+1}}, \frac{z}{a^{k+1}}, \frac{w}{a^{k+1}}\right)-a^{10 k} f\left(\frac{x}{a^{k}}, \frac{y}{a^{k}}, \frac{z}{a^{k}}, \frac{w}{a^{k}}\right),|a|^{10 k} t\right) \leq N\left(x, y, z, w,|\alpha|^{k+1} t\right),
\end{array}\right.
$$

for all $x, y, z, w \in X, t>0$ and an integer $k \geq 0$. By replacing $t$ by $\frac{t}{|\alpha|^{k+1}}$, we get

$$
\left\{\begin{array}{l}
\mathcal{F}\left(a^{10(k+1)} f\left(\frac{x}{a^{k+1}}, \frac{y}{a^{k+1}}, \frac{z}{a^{k+1}}, \frac{w}{a^{k+1}}\right)-a^{10 k} f\left(\frac{x}{a^{k}}, \frac{y}{a^{k}}, \frac{z}{a^{k}}, \frac{w}{a^{k}}\right), \frac{|a|^{10 k}}{|\alpha|^{k+1}} t\right) \geq M(x, y, z, w, t) \\
\mathcal{P}\left(a^{10(k+1)} f\left(\frac{x}{a^{k+1}}, \frac{y}{a^{k+1}}, \frac{z}{a^{k+1}}, \frac{w}{a^{k+1}}\right)-a^{10 k} f\left(\frac{x}{a^{k}}, \frac{y}{a^{k}}, \frac{z}{a^{k}}, \frac{w}{a^{k}}\right), \frac{|a|^{10 k}}{|\alpha|^{k+1}} t\right) \leq N(x, y, z, w, t) .
\end{array}\right.
$$

It follows from

$$
\begin{aligned}
a^{10 n} f\left(\frac{x}{a^{n}}, \frac{y}{a^{n}}, \frac{z}{a^{n}}, \frac{w}{a^{n}}\right)-f(x, y, z, w) & \\
& =\sum_{k=0}^{n-1}\left(a^{10(k+1)} f\left(\frac{x}{a^{k+1}}, \frac{y}{a^{k+1}}, \frac{z}{a^{k+1}}, \frac{w}{a^{k+1}}\right)-a^{10 k} f\left(\frac{x}{a^{k}}, \frac{y}{a^{k}}, \frac{z}{a^{k}}, \frac{w}{a^{k}}\right)\right)
\end{aligned}
$$

and (3.14) that

$$
\begin{aligned}
\mathcal{F}\left(a ^ { 1 0 n } f \left(\frac{x}{a^{n}}\right.\right. & \left.\left., \frac{y}{a^{n}}, \frac{z}{a^{n}}, \frac{w}{a^{n}}\right)-f(x, y, z, w), \sum_{k=0}^{n-1} \frac{|a|^{10 k}}{|\alpha|^{k+1}} t\right) \\
& \geq \prod_{k=0}^{n-1} \mathcal{F}\left(a^{10(k+1)} f\left(\frac{x}{a^{k+1}}, \frac{y}{a^{k+1}}, \frac{z}{a^{k+1}}, \frac{w}{a^{k+1}}\right)-a^{10 k} f\left(\frac{x}{a^{k}}, \frac{y}{a^{k}}, \frac{z}{a^{k}}, \frac{w}{a^{k}}\right), \frac{|a|^{10 k}}{|\alpha|^{k+1}} t\right) \\
& \geq M(x, y, z, w, t) \\
\mathcal{P}\left(a ^ { 1 0 n } f \left(\frac{x}{a^{n}},\right.\right. & \left.\left.\frac{y}{a^{n}}, \frac{z}{a^{n}}, \frac{w}{a^{n}}\right)-f(x, y, z, w), \sum_{k=0}^{n-1} \frac{|a|^{10 k}}{|\alpha|^{k+1}} t\right) \\
& \leq \coprod_{k=0}^{n-1} \mathcal{P}\left(a^{10(k+1)} f\left(\frac{x}{a^{k+1}}, \frac{y}{a^{k+1}}, \frac{z}{a^{k+1}}, \frac{w}{a^{k+1}}\right)-a^{10 k} f\left(\frac{x}{a^{k}}, \frac{y}{a^{k}}, \frac{z}{a^{k}}, \frac{w}{a^{k}}\right), \frac{|a|^{10 k}}{|\alpha|^{k+1}} t\right) \\
& \leq N(x, y, z, w, t),
\end{aligned}
$$

for all $x, y, z, w \in X, t>0$ and $n>0$, where $\prod_{k=1}^{n} \varepsilon_{k}=\varepsilon_{1} * \varepsilon_{2} * \ldots * \varepsilon_{n}$ and $\coprod_{k=1}^{n} \varepsilon_{k}=\varepsilon_{1} \diamond \varepsilon_{2} \diamond \ldots \diamond \varepsilon_{n}$. Replacing $x, y, z, w$ by $\frac{x}{a^{k}}, \frac{y}{a^{k}}, \frac{z}{a^{k}}, \frac{w}{a^{k}}$ respectively in the last inequalities, we have 


$$
\begin{aligned}
\mathcal{F}\left(a^{10(n+p)} f(\right. & \left.\left.\frac{x}{a^{n+p}}, \frac{y}{a^{n+p}}, \frac{z}{a^{n+p}}, \frac{w}{a^{n+p}}\right)-a^{10 p} f\left(\frac{x}{a^{p}}, \frac{y}{a^{p}}, \frac{z}{a^{p}}, \frac{w}{a^{p}}\right), \sum_{k=0}^{n-1} \frac{|a|^{10(k+p)}}{|\alpha|^{k+1}} t\right) \\
& \geq M\left(x, y, z, w,|\alpha|^{p} t\right) \\
\mathcal{P}\left(a^{10(n+p)} f(\right. & \left.\left.\frac{x}{a^{n+p}}, \frac{y}{a^{n+p}}, \frac{z}{a^{n+p}}, \frac{w}{a^{n+p}}\right)-a^{10 p} f\left(\frac{x}{a^{p}}, \frac{y}{a^{p}}, \frac{z}{a^{p}}, \frac{w}{a^{p}}\right), \sum_{k=0}^{n-1} \frac{|a|^{10(k+p)}}{|\alpha|^{k+1}} t\right) \\
& \leq N\left(x, y, z, w,|\alpha|^{p} t\right) .
\end{aligned}
$$

Whence

$$
\begin{aligned}
\mathcal{F}\left(a^{10(n+p)} f(\right. & \left.\frac{x}{a^{n+p}}, \frac{y}{a^{n+p}}, \frac{z}{a^{n+p}}, \frac{w}{a^{n+p}}\right)-a^{10 p} f\left(\frac{x}{a^{p}}, \frac{y}{a^{p}}, \frac{z}{a^{p}}, \frac{w}{a^{p}}\right), \sum_{k=0}^{n-1} \frac{|a|^{10(k+p)}}{\left.|\alpha|^{k+p+1} t\right)} \\
& \geq M(x, y, z, w, t) \\
\mathcal{P}\left(a^{10(n+p)} f(\right. & \left.\left.\frac{x}{a^{n+p}}, \frac{y}{a^{n+p}}, \frac{z}{a^{n+p}}, \frac{w}{a^{n+p}}\right)-a^{10 p} f\left(\frac{x}{a^{p}}, \frac{y}{a^{p}}, \frac{z}{a^{p}}, \frac{w}{a^{p}}\right), \sum_{k=0}^{n-1} \frac{|a|^{10(k+p)}}{|\alpha|^{k+p+1}} t\right) \\
& \leq N(x, y, z, w, t)
\end{aligned}
$$

for all $x, y, z, w \in X, t>0, n>0$ and $p \geq 0$. Hence,

$$
\left\{\begin{aligned}
\mathcal{F}\left(a^{10(n+p)} f\left(\frac{x}{a^{n+p}}, \frac{y}{a^{n+p}}, \frac{z}{a^{n+p}}, \frac{w}{a^{n+p}}\right)\right. & \left.-f\left(\frac{x}{a^{p}}, \frac{y}{a^{p}}, \frac{z}{a^{p}}, \frac{w}{a^{p}}\right), t\right) \\
& \geq M\left(x, y, z, w, \frac{t}{\sum_{k=p}^{n+p-1} \frac{|a|^{10 k}}{|\alpha|^{k+1}}}\right) \\
\mathcal{P}\left(a^{10(n+p)} f\left(\frac{x}{a^{n+p}}, \frac{y}{a^{n+p}}, \frac{z}{a^{n+p}}, \frac{w}{a^{n+p}}\right)\right. & \left.-f\left(\frac{x}{a^{p}}, \frac{y}{a^{p}}, \frac{z}{a^{p}}, \frac{w}{a^{p}}\right), t\right) \\
& \leq N\left(x, y, z, w, \frac{t}{\sum_{k=p}^{n+p-1} \frac{|a|^{10 k}}{|\alpha|^{k+1}}}\right) .
\end{aligned}\right.
$$

Since $|\alpha|>|a|^{10}$, we have $\sum_{k=0}^{\infty} \frac{|a|^{10 k}}{|\alpha|^{k+1}}<\infty$. This shows that $\left(a^{10 n} f\left(\frac{x}{a^{n}}, \frac{y}{a^{n}}, \frac{z}{a^{n}}, \frac{w}{a^{n}}\right)\right)$ is a Cauchy sequence in non-Archimedean intuitionistic fuzzy Banach space $(Y, \mathcal{F}, \mathcal{P})$ and so it converges to some point $D(x, y, z, w) \in Y$. Thus, we define a mapping $D: X \times X \times X \times X \rightarrow Y$ such that

$$
D(x, y, z, w)=(\mathcal{F}, \mathcal{P})-\lim _{n \rightarrow \infty} a^{10 n} f\left(\frac{x}{a^{n}}, \frac{y}{a^{n}}, \frac{z}{a^{n}}, \frac{w}{a^{n}}\right) .
$$

Hence for all $x, y, z, w \in X$ and $t>0$, we have

$$
\begin{aligned}
& \lim _{n \rightarrow \infty} \mathcal{F}\left(a^{10 n} f\left(\frac{x}{a^{n}}, \frac{y}{a^{n}}, \frac{z}{a^{n}}, \frac{w}{a^{n}}\right)-D(x, y, z, w)\right)=1 \\
& \lim _{n \rightarrow \infty} \mathcal{P}\left(a^{10 n} f\left(\frac{x}{a^{n}}, \frac{y}{a^{n}}, \frac{z}{a^{n}}, \frac{w}{a^{n}}\right)-D(x, y, z, w)\right)=0 .
\end{aligned}
$$


Moreover, if we put $p=0$ in (3.15), we get

$$
\begin{aligned}
& \mathcal{F}\left(a^{10 n} f\left(\frac{x}{a^{n}}, \frac{y}{a^{n}}, \frac{z}{a^{n}}, \frac{w}{a^{n}}\right)-f(x, y, z, w), t\right) \geq M\left(x, y, z, w, \frac{t}{\sum_{k=0}^{n-1} \frac{|a|^{10 k}}{|\alpha|^{k+1}}}\right) \\
& \mathcal{P}\left(a^{10 n} f\left(\frac{x}{a^{n}}, \frac{y}{a^{n}}, \frac{z}{a^{n}}, \frac{w}{a^{n}}\right)-f(x, y, z, w), t\right) \leq N\left(x, y, z, w, \frac{t}{\sum_{k=0}^{n-1} \frac{|a|^{10 k}}{|\alpha|^{k+1}}}\right)
\end{aligned}
$$

for all $x, y, z, w \in X, t>0$ and $n>0$. Therefore,

$$
\begin{aligned}
& \mathcal{F}(D(x, y, z, w)-f(x, y, z, w), t) \geq \mathcal{F}\left(D(x, y, z, w)-a^{10 n} f\left(\frac{x}{a^{n}}, \frac{y}{a^{n}}, \frac{z}{a^{n}}, \frac{w}{a^{n}}\right), t\right) \\
& * \mathcal{F}\left(a^{10 n} f\left(\frac{x}{a^{n}}, \frac{y}{a^{n}}, \frac{z}{a^{n}}, \frac{w}{a^{n}}\right)-f(x, y, z, w), t\right) \geq M\left(x, y, z, w, \frac{t}{\sum_{k=0}^{n-1} \frac{|a|^{10 k}}{|\alpha|^{k+1}}}\right) \\
& \mathcal{P}(D(x, y, z, w)-f(x, y, z, w), t) \geq \mathcal{P}\left(D(x, y, z, w)-a^{10 n} f\left(\frac{x}{a^{n}}, \frac{y}{a^{n}}, \frac{z}{a^{n}}, \frac{w}{a^{n}}\right), t\right) \\
& \diamond \mathcal{P}\left(a^{10 n} f\left(\frac{x}{a^{n}}, \frac{y}{a^{n}}, \frac{z}{a^{n}}, \frac{w}{a^{n}}\right)-f(x, y, z, w), t\right) \leq N\left(x, y, z, w, \frac{t}{\sum_{k=0}^{n-1} \frac{|a|^{10 k}}{|\alpha|^{k+1}}}\right)
\end{aligned}
$$

for all $x, y, z, w \in X, t>0$ and $n>0$. Letting $n \rightarrow \infty$ in the above inequalities, we

$$
\begin{aligned}
& \mathcal{F}(D(x, y, z, w)-f(x, y, z, w), t) \geq M\left(x, y, z, w,\left(|\alpha|-|a|^{10}\right) t\right) \\
& \mathcal{P}(D(x, y, z, w)-f(x, y, z, w), t) \geq M\left(x, y, z, w,\left(|\alpha|-|a|^{10}\right) t\right) .
\end{aligned}
$$

Hence, $D$ satisfies (3.6). Let $x, y, z, w, x_{1}, x_{2}, y_{1}, y_{2}, z_{1}, z_{2}, w_{1}, w_{2} \in X$, then

$$
\begin{aligned}
& \left\{\begin{aligned}
\mathcal{F} & \left(D\left(a x_{1}+b x_{2}, y, z, w\right)+D\left(a x_{1}-b x_{2}, y, z, w\right)-2 a D\left(x_{1}, y, z, w\right), t\right) \\
& \geq \mathcal{F}^{\prime}\left(\varphi_{1}\left(x_{1}, x_{2}, y, z, w\right), \frac{|\alpha|^{n}}{|a|^{10 n}} t\right) \\
& \mathcal{P}\left(D\left(a x_{1}+b x_{2}, y, z, w\right)+D\left(a x_{1}-b x_{2}, y, z, w\right)-2 a D\left(x_{1}, y, z, w\right), t\right) \\
& \leq \mathcal{P}^{\prime}\left(\varphi_{1}\left(x_{1}, x_{2}, y, z, w\right), \frac{|\alpha|^{n}}{|a|^{10 n}} t\right)
\end{aligned}\right. \\
& \left\{\begin{aligned}
& \mathcal{F}\left(D\left(x, a y_{1}+b y_{2}, z, w\right)+D\left(x, a y_{1}-b y_{2}, z, w\right)-2 a^{2} D\left(x, y_{1}, z, w\right)-2 b^{2} D\left(x, y_{2}, z, w\right), t\right) \\
\geq & \mathcal{F}^{\prime}\left(\varphi_{2}\left(x, y_{1}, y_{2}, z, w\right), \frac{|\alpha|^{n}}{|a|^{10 n}} t\right) \\
& \mathcal{P}\left(D\left(x, a y_{1}+b y_{2}, z, w\right)+D\left(x, a y_{1}-b y_{2}, z, w\right)-2 a^{2} D\left(x, y_{1}, z, w\right)-2 b^{2} D\left(x, y_{2}, z, w\right), t\right) \\
\leq & \mathcal{P}^{\prime}\left(\varphi_{2}\left(x, y_{1}, y_{2}, z, w\right), \frac{|\alpha|^{n}}{|a|^{10 n}} t\right),
\end{aligned}\right.
\end{aligned}
$$




$$
\left\{\begin{array}{l}
\mathcal{F}\left(D\left(x, y, a z_{1}+b z_{2}, w\right)+D\left(x, y, a z_{1}-b z_{2}, w\right)-a b^{2}\left(D\left(x, y, z_{1}+z_{2}, w\right)+D\left(x, y, z_{1}-z_{2},, w\right)\right)\right. \\
\left.-2 a\left(a^{2}-b^{2}\right) D\left(x, y, z_{1}, w\right), t\right) \geq \mathcal{F}^{\prime}\left(\varphi_{3}\left(x, y, z_{1}, z_{2}, w\right), \frac{|\alpha|^{n}}{|a|^{10 n}} t\right) \\
\mathcal{P}\left(D\left(x, y, a z_{1}+b z_{2}, w\right)+D\left(x, y, a z_{1}-b z_{2}, w\right)-a b^{2}\left(D\left(x, y, z_{1}+z_{2}, w\right)+D\left(x, y, z_{1}-z_{2},, w\right)\right)\right. \\
\left.-2 a\left(a^{2}-b^{2}\right) D\left(x, y, z_{1}, w\right), t\right) \leq \mathcal{P}^{\prime}\left(\varphi_{3}\left(x, y, z_{1}, z_{2}, w\right), \frac{|\alpha|^{n}}{|a|^{10 n}} t\right)
\end{array}\right.
$$

and

$$
\left\{\begin{array}{l}
\mathcal{F}\left(D\left(x, y, z, a w_{1}+b w_{2}\right)+D\left(x, y, z, a w_{1}-b w_{2}\right)-a^{2} b^{2}\left(D\left(x, y, z, w_{1}+w_{2}\right)+D\left(x, y, z, w_{1}-w_{2}\right)\right)\right. \\
\left.\left.-2 a^{2}\left(a^{2}-b^{2}\right) D\left(x, y, z, w_{1}\right)+2 b^{2}\left(a^{2}-b^{2}\right) D\left(x, y, z, w_{2}\right), t\right) \geq \mathcal{F}^{\prime}\left(\varphi_{4}\left(x, y, z, w_{1}, w_{2}\right), \frac{|\alpha|^{n}}{|a|^{10 n}} t\right),(3.1)+w^{2}\right) \\
\mathcal{P}\left(D\left(x, y, z, a w_{1}+b w_{2}\right)+D\left(x, y, z, a w_{1}-b w_{2}\right)-a^{2} b^{2}\left(D\left(x, y, z, w_{1}+w_{2}\right)+D\left(x, y, z, w_{1}-w_{2}\right)\right)\right. \\
\left.-2 a^{2}\left(a^{2}-b^{2}\right) D\left(x, y, z, w_{1}\right)+2 b^{2}\left(a^{2}-b^{2}\right) D\left(x, y, z, w_{2}\right), t\right) \leq \mathcal{P}^{\prime}\left(\varphi_{4}\left(x, y, z, w_{1}, w_{2}\right), \frac{|\alpha|^{n}}{|a|^{10 n}} t\right) .
\end{array}\right.
$$

Letting $n \rightarrow \infty$ in (3.16), (3.17), (3.18) and (3.19), we get

$\mathcal{F}\left(D\left(a x_{1}+b x_{2}, y, z, w\right)+D\left(a x_{1}-b x_{2}, y, z, w\right)-2 a D\left(x_{1}, y, z, w\right), t\right)=1$,

$\mathcal{F}\left(D\left(x, a y_{1}+b y_{2}, z, w\right)+D\left(x, a y_{1}-b y_{2}, z, w\right)-2 a^{2} D\left(x, y_{1}, z, w\right)-2 b^{2} D\left(x, y_{2}, z, w\right), t\right)=1$

$\mathcal{F}\left(D\left(x, y, a z_{1}+b z_{2}, w\right)+D\left(x, y, a z_{1}-b z_{2}, w\right)-a b^{2}\left(D\left(x, y, z_{1}+z_{2}, w\right)+D\left(x, y, z_{1}-z_{2},, w\right)\right)\right.$ $\left.-2 a\left(a^{2}-b^{2}\right) D\left(x, y, z_{1}, w\right), t\right)=1$,

$\mathcal{F}\left(D\left(x, y, z, a w_{1}+b w_{2}\right)+D\left(x, y, z, a w_{1}-b w_{2}\right)-a^{2} b^{2}\left(D\left(x, y, z, w_{1}+w_{2}\right)+D\left(x, y, z, w_{1}-w_{2}\right)\right)\right.$ $\left.-2 a^{2}\left(a^{2}-b^{2}\right) D\left(x, y, z, w_{1}\right)+2 b^{2}\left(a^{2}-b^{2}\right) D\left(x, y, z, w_{2}\right), t\right)=1$,

and similarly, we obtain

$\mathcal{P}\left(D\left(a x_{1}+b x_{2}, y, z, w\right)+D\left(a x_{1}-b x_{2}, y, z, w\right)-2 a D\left(x_{1}, y, z, w\right), t\right)=0$,

$\mathcal{P}\left(D\left(x, a y_{1}+b y_{2}, z, w\right)+D\left(x, a y_{1}-b y_{2}, z, w\right)-2 a^{2} D\left(x, y_{1}, z, w\right)-2 b^{2} D\left(x, y_{2}, z, w\right), t\right)=0$

$\mathcal{P}\left(D\left(x, y, a z_{1}+b z_{2}, w\right)+D\left(x, y, a z_{1}-b z_{2}, w\right)-a b^{2}\left(D\left(x, y, z_{1}+z_{2}, w\right)+D\left(x, y, z_{1}-z_{2},, w\right)\right)\right.$ $\left.-2 a\left(a^{2}-b^{2}\right) D\left(x, y, z_{1}, w\right), t\right)=0$,

$\mathcal{P}\left(D\left(x, y, z, a w_{1}+b w_{2}\right)+D\left(x, y, z, a w_{1}-b w_{2}\right)-a^{2} b^{2}\left(D\left(x, y, z, w_{1}+w_{2}\right)+D\left(x, y, z, w_{1}-w_{2}\right)\right)\right.$ $\left.-2 a^{2}\left(a^{2}-b^{2}\right) D\left(x, y, z, w_{1}\right)+2 b^{2}\left(a^{2}-b^{2}\right) D\left(x, y, z, w_{2}\right), t\right)=0$,

for all $x, y, z, w, x_{1}, x_{2}, y_{1}, y_{2}, z_{1}, z_{2}, w_{1}, w_{2} \in X$ and $t>0$. This means that $D$ satisfies (1.1) and so it is dectic mapping. To prove the uniqueness of $D$, assume that $D^{\prime}$ be another dectic mapping from $X \times X \times$ $X \times X$ into $Y$, which satisfies (3.6). For all $x, y, z, w \in X$, all $n>0$, clearly $D(x, y, z, w)=$ $a^{10 n} D\left(\frac{x}{a^{n}}, \frac{y}{a^{n}}, \frac{z}{a^{n}}, \frac{w}{a^{n}}\right)$ and $D^{\prime}(x, y, z, w)=a^{10 n} D^{\prime}\left(\frac{x}{a^{n}}, \frac{y}{a^{n}}, \frac{z}{a^{n}}, \frac{w}{a^{n}}\right)$. It follows from (3.6) that 


$$
\left\{\begin{array}{l}
\mathcal{F}\left(D(x, y, z, w)-D^{\prime}(x, y, z, w), t\right) \geq M\left(x, y, z, w, \frac{|\alpha|^{n}\left(|\alpha|-|a|^{10}\right)}{|a|^{10 n}} t\right) \\
\mathcal{P}\left(D(x, y, z, w)-D^{\prime}(x, y, z, w), t\right) \leq N\left(x, y, z, w, \frac{|\alpha|^{n}\left(|\alpha|-|a|^{10}\right)}{|a|^{10 n}} t\right) .
\end{array}\right.
$$

We see that the right-hand sides of (3.20) tending to 1 and 0 , respectively, as $n \rightarrow \infty$. Therefore, $\mathcal{F}\left(D(x, y, z, w)-D^{\prime}(x, y, z, w), t\right)=1$ and $\mathcal{P}\left(D(x, y, z, w)-D^{\prime}(x, y, z, w), t\right)=0$, for all $x, y, z, w \in$ $X$ and $t>0$. Hence, $D(x, y, z, w)=D^{\prime}(x, y, z, w)$.

Theorem 3.1. Let $X$ be a linear space over a non-Archimedean field $K$ and let $\left(Z, \mathcal{F}^{\prime}, \mathcal{P}^{\prime}\right)$ be a nonArchimedean IFN space. Suppose that $\varphi_{i}: X \times X \times X \times X \times X \rightarrow Z$ for $i \in I$ be functions such that $|a|<1$ is far from zero and for some real number $\alpha$ with $0<|\alpha|<|a|^{10}$,

$$
\left\{\begin{array}{l}
\mathcal{F}^{\prime}\left(\varphi_{i}(a x, a y, a z, a w, t) \geq \mathcal{F}^{\prime}\left(|\alpha| \varphi_{i}(x, y, z, w, v), t\right)\right. \\
\mathcal{P}^{\prime}\left(\varphi_{i}(a x, a y, a z, a w, t) \leq \mathcal{P}^{\prime}\left(|\alpha| \varphi_{i}(x, y, z, w, v), t\right)\right.
\end{array}\right.
$$

for all $x, y, z, w, v \in X, t>0$ and $i \in I$. Let $f$ be a mapping from $X \times X \times X \times X$ to a nonArchimedean intuitionistic fuzzy Banach space $(Y, \mathcal{F}, \mathcal{P})$ such that $f(x, 0, z, w)=0, f(x, y, z, 0)=0$ for all $x, y, z, w \in X$, and satisfies (3.2), (3.3), (3.4) and (3.5). Then there exists a unique dectic mapping $D: X \times X \times X \times X \rightarrow Y$ such that

$$
\left\{\begin{array}{l}
\mathcal{F}(f(x, y, z, w)-D(x, y, z, w), t) \geq M\left(x, y, z, w,\left(|a|^{10}-|\alpha|\right) t\right) \\
\mathcal{P}(f(x, y, z, w)-D(x, y, z, w), t) \leq N\left(x, y, z, w,\left(|a|^{10}-|\alpha|\right) t\right)
\end{array}\right.
$$

for all $x, y, z, w \in X$ and $t>0$, where $M(x, y, z, w, t)$ and $N(x, y, z, w, t)$ are defined as in Theorem 3.1.

Proof. The proof can be done by the same way as in Theorem 3.1.

\section{Non-Archimedean intuitionistic fuzzy continuity}

Recently, the intuitionistic fuzzy continuity is discussed in [19]. In this section, we establish nonArchimedean intuitionistic fuzzy continuity of continuous mappings satisfying (1.1) approximately.

Definition 4.1. Let $g: \mathbb{R} \rightarrow X$ be a mapping, where $\mathbb{R}$ is endowed with the Euclidean topology and $X$ is a non-Archimedean intuitionistic fuzzy normed space equipped with non-Archimedean intuitionistic fuzzy norm $(\mathcal{F}, \mathcal{P})$. Then, $g$ is called non-Archimedean intuitionistic fuzzy continuous at a points $r_{0} \in \mathbb{R}$ if for all $\epsilon>0$ and $0<\rho<1$ there exists some $\delta>0$ such that for each $r$ with $0<\left|r-r_{0}\right|<\delta$,

$$
\mathcal{F}\left(g(r x)-g\left(r_{0} x\right), \epsilon\right) \geq \rho, \quad \mathcal{P}\left(g(r x)-g\left(r_{0} x\right), \epsilon\right) \leq 1-\rho .
$$

Theorem 4.2. Let $X$ be a non-Archimedean space and let $\left(Z, \mathcal{F}^{\prime}, \mathcal{P}^{\prime}\right)$ be a non-Archimedean IFN space. Let $p>10$ and $z_{0} \in Z$. Let $f$ be a mapping from $X \times X \times X \times X$ to a non- Archimedean intuitionistic fuzzy Banach space $(Y, \mathcal{F}, \mathcal{P})$ such that $f(x, 0, z, w)=0, f(x, y, z, 0)=0$ for all $x, y, z, w \in X$, and satisfying 

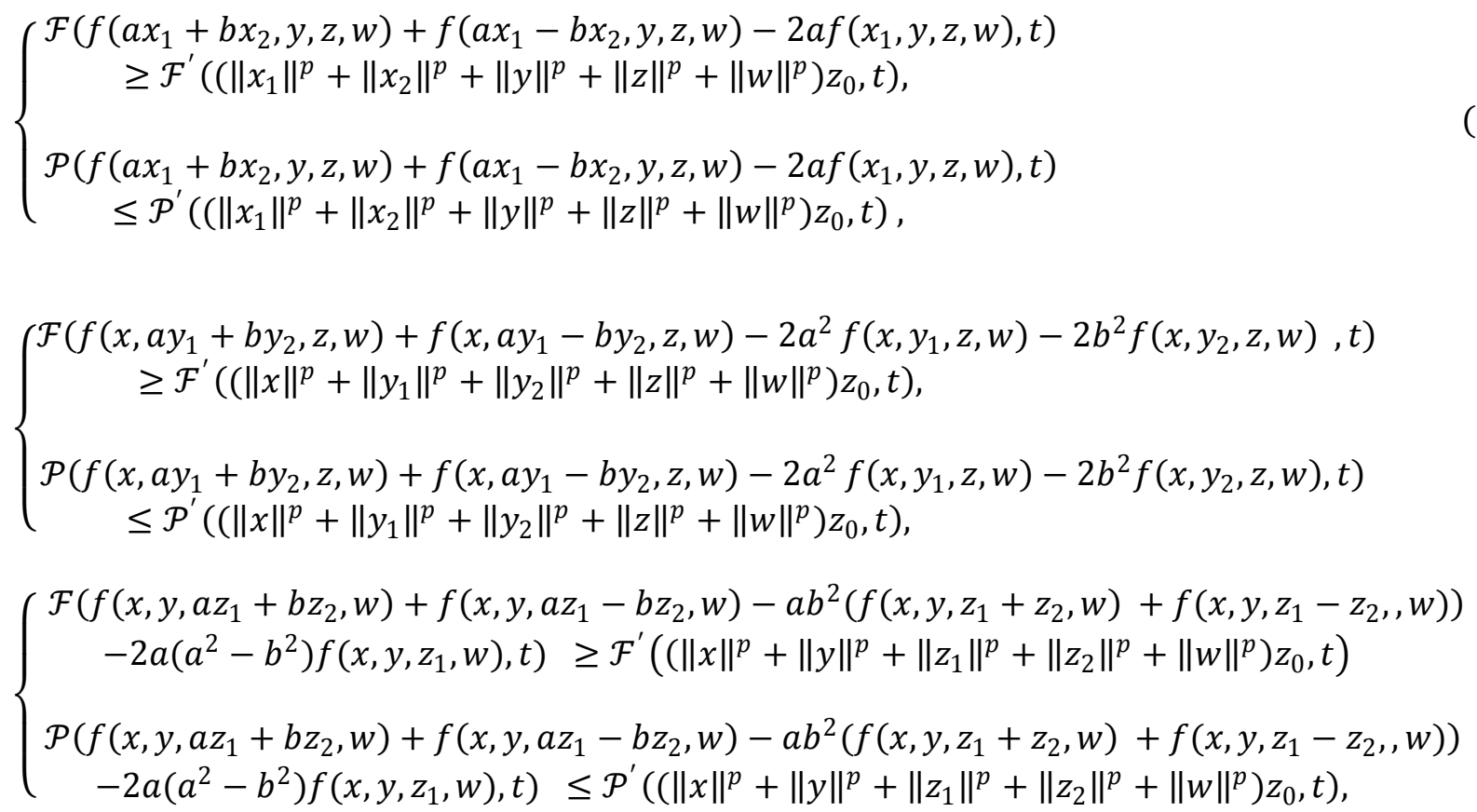

and

$$
\left\{\begin{array}{c}
\mathcal{F}\left(f\left(x, y, z, a w_{1}+b w_{2}\right)+f\left(x, y, z, a w_{1}-b w_{2}\right)-a^{2} b^{2}\left(f\left(x, y, z, w_{1}+w_{2}\right)+f\left(x, y, z, w_{1}-w_{2}\right)\right)\right. \\
\left.-2 a^{2}\left(a^{2}-b^{2}\right) f\left(x, y, z, w_{1}\right)+2 b^{2}\left(a^{2}-b^{2}\right) f\left(x, y, z, w_{2}\right), t\right) \\
\geq \mathcal{F}^{\prime}\left(\left(\|x\|^{p}+\|y\|^{p}+\|z\|^{p}+\left\|w_{1}\right\|^{p}+\left\|w_{2}\right\|^{p}\right) z_{0}, t\right) \\
\mathcal{P}\left(f\left(x, y, z, a w_{1}+b w_{2}\right)+f\left(x, y, z, a w_{1}-b w_{2}\right)-a^{2} b^{2}\left(f\left(x, y, z, w_{1}+w_{2}\right)+f\left(x, y, z, w_{1}-w_{2}\right)\right)\right. \\
\left.-2 a^{2}\left(a^{2}-b^{2}\right) f\left(x, y, z, w_{1}\right)+2 b^{2}\left(a^{2}-b^{2}\right) f\left(x, y, z, w_{2}\right), t\right) \\
\leq \mathcal{P}^{\prime}\left(\left(\|x\|^{p}+\|y\|^{p}+\|z\|^{p}+\left\|w_{1}\right\|^{p}+\left\|w_{2}\right\|^{p}\right) z_{0}, t\right)
\end{array}\right.
$$

for all $x, y, z, w, x_{1}, x_{2}, y_{1}, y_{2}, z_{1}, z_{2}, w_{1}, w_{2} \in X$ and all $t>0$. Then there exists a unique dectic mapping $D: X \times X \times X \times X \rightarrow Y$ such that

$$
\left\{\begin{aligned}
\mathcal{F}(f(x, y, z, w)-D(x, y, z, w), t) \\
\geq \mathcal{F}^{\prime}\left(\left(\|x\|^{p}+\|y\|^{p}+\|z\|^{p}+\|w\|^{p}\right) z_{0},|2||a|^{-9}\left(|a|^{10}-|a|^{p}\right) t\right) \\
* \mathcal{F}^{\prime}\left(\left(\|x\|^{p}+\|y\|^{p}+\|z\|^{p}+\|w\|^{p}\right) z_{0},|2||a|^{-7}\left(|a|^{10}-|a|^{p}\right) t\right) \\
* \mathcal{F}^{\prime}\left(\left(\|x\|^{p}+\|y\|^{p}+\|z\|^{p}+\|w\|^{p}\right) z_{0},|2||a|^{-4}\left(|a|^{10}-|a|^{p}\right) t\right) \\
\quad * \mathcal{F}^{\prime}\left(\left(\|x\|^{p}+\|y\|^{p}+\|z\|^{p}+\|w\|^{p}\right) z_{0},|2|\left(|a|^{10}-|a|^{p}\right) t\right) \\
\mathcal{P}(f(x, y, z, w)-D(x, y, z, w), t) \\
\leq \mathcal{P}^{\prime}\left(\left(\|x\|^{p}+\|y\|^{p}+\|z\|^{p}+\|w\|^{p}\right) z_{0},|2||a|^{-9}\left(|a|^{10}-|a|^{p}\right) t\right) \\
\diamond \mathcal{P}^{\prime}\left(\left(\|x\|^{p}+\|y\|^{p}+\|z\|^{p}+\|w\|^{p}\right) z_{0},|2||a|^{-7}\left(|a|^{10}-|a|^{p}\right) t\right) \\
\diamond \mathcal{P}^{\prime}\left(\left(\|x\|^{p}+\|y\|^{p}+\|z\|^{p}+\|w\|^{p}\right) z_{0},|2||a|^{-4}\left(|a|^{10}-|a|^{p}\right) t\right) \\
\Delta \mathcal{P}^{\prime}\left(\left(\|x\|^{p}+\|y\|^{p}+\|z\|^{p}+\|w\|^{p}\right) z_{0},|2|\left(|a|^{10}-|a|^{p}\right) t\right)
\end{aligned}\right.
$$


for all $x, y, z, w \in X$ and all $t>0$. Furthermore, if the mapping $g: \mathbb{R} \rightarrow Y$ defined by $g(r):=$ $\frac{f\left(a^{n} r x, a^{n} r y, a^{n} r z, a^{n} r w\right)}{a^{10 n}}$ is non-Archimedean intuitionistic fuzzy continuous for some $x, y, z, w \in X$ and all $n \in \mathbb{N}$ then the mapping $r \rightarrow D(r x, r y, r z, r w)$ from $\mathbb{R}$ to $Y$ is non-Archimedean intuitionistic fuzzy continuous.

Proof. Define $\varphi_{i}: X \times X \times X \times X \rightarrow Z$ by $\varphi_{i}(x, y, z, w)=\left(\|x\|^{p}+\|y\|^{p}+\|z\|^{p}+\|w\|^{p}+\|v\|^{p}\right) z_{0}$ for all $x, y, z, w, v \in X$ and all $i \in I$. Existence and uniqueness of the dectic mapping $D$ satisfying (4.5) are deduced from Theorem 3.2. Note that, for all $x, y, z, w \in X$, all $t>0$ and $n \in \mathbb{N}$ we have

$$
\left\{\begin{aligned}
\mathcal{F}(D(x, y, z, w) & \left.-\frac{f\left(a^{n} x, a^{n} y, a^{n} z, a^{n} w\right)}{a^{10 n}}, t\right) \\
\geq & \mathcal{F}^{\prime}\left(\left(\|x\|^{p}+\|y\|^{p}+\|z\|^{p}+\|w\|^{p}\right) z_{0}, \frac{|2 \| a|^{-9}|a|^{10 n}\left(|a|^{10}-|a|^{p}\right)}{|a|^{n p}} t\right) \\
& * \mathcal{F}^{\prime}\left(\left(\|a x\|^{p}+\|y\|^{p}+\|z\|^{p}+\|w\|^{p}\right) z_{0}, \frac{|2 \| a|^{-7}|a|^{10 n}\left(|a|^{10}-|a|^{p}\right)}{|a|^{n p}} t\right) \\
& * \mathcal{F}^{\prime}\left(\left(\|a x\|^{p}+\|a y\|^{p}+\|z\|^{p}+\|w\|^{p}\right) z_{0}, \frac{|2||a|^{-4}|a|^{10 n}\left(|a|^{10}-|a|^{p}\right)}{|a|^{n p}} t\right) \\
& * \mathcal{F}^{\prime}\left(\left(\|a x\|^{p}+\|a y\|^{p}+\|a z\|^{p}+\|w\|^{p}\right) z_{0}, \frac{|2||a|^{10 n}\left(|a|^{10}-|a|^{p}\right)}{|a|^{n p}} t\right) \\
\mathcal{P}(f(x, y, z, w) & \left.-\frac{f\left(a^{n} x, a^{n} y, a^{n} z, a^{n} w\right)}{a^{10 n}}, t\right) \\
& \leq \mathcal{P}^{\prime}\left(\left(\|x\|^{p}+\|y\|^{p}+\|z\|^{p}+\|w\|^{p}\right) z_{0}, \frac{\left.|2 \|| a\right|^{-9}|a|^{10 n}\left(|a|^{10}-|a|^{p}\right)}{|a|^{n p}} t\right) \\
& \diamond \mathcal{P}^{\prime}\left(\left(\|a x\|^{p}+\|y\|^{p}+\|z\|^{p}+\|w\|^{p}\right) z_{0}, \frac{\left.|2 \|| a\right|^{-7}|a|^{10 n}\left(|a|^{10}-|a|^{p}\right)}{|a|^{n p}} t\right) \\
& \diamond \mathcal{P}^{\prime}\left(\left(\|a x\|^{p}+\|a y\|^{p}+\|z\|^{p}+\|w\|^{p}\right) z_{0}, \frac{\left.|2 \|| a\right|^{-4}|a|^{10 n}\left(|a|^{10}-|a|^{p}\right)}{|a|^{n p}} t\right) \\
& \diamond \mathcal{P}^{\prime}\left(\left(\|a x\|^{p}+\|a y\|^{p}+\|a z\|^{p}+\|w\|^{p}\right) z_{0}, \frac{|2||a|^{10 n}\left(|a|^{10}-|a|^{p}\right)}{|a|^{n p}} t\right)
\end{aligned}\right.
$$

Fix $x, y, z, w, v \in X$ and $r_{0} \in \mathbb{R}$. Given $\epsilon>0$ and $0<\rho<1$. From (4.6) it is follows that 


$$
\left\{\begin{aligned}
\mathcal{F}(D(r x, r y, r z, r w) & \left.-\frac{f\left(a^{n} r x, a^{n} r y, a^{n} r z, a^{n} r w\right)}{a^{10 n}}, t\right) \\
\geq & \mathcal{F}^{\prime}\left(\left(\|x\|^{p}+\|y\|^{p}+\|z\|^{p}+\|w\|^{p}\right) z_{0}, \frac{|2||a|^{-9}|a|^{10 n}\left(|a|^{10}-|a|^{p}\right)}{\left(1+r_{0}\right)^{p}|a|^{n p}} t\right) \\
& * \mathcal{F}^{\prime}\left(\left(\|a x\|^{p}+\|y\|^{p}+\|z\|^{p}+\|w\|^{p}\right) z_{0}, \frac{|2||a|^{-7}|a|^{10 n}\left(|a|^{10}-|a|^{p}\right)}{\left(1+r_{0}\right)^{p}|a|^{n p}} t\right) \\
& * \mathcal{F}^{\prime}\left(\left(\|a x\|^{p}+\|a y\|^{p}+\|z\|^{p}+\|w\|^{p}\right) z_{0}, \frac{|2||a|^{-4}|a|^{10 n}\left(|a|^{10}-|a|^{p}\right)}{\left.\left(1+r_{0}\right)^{p}|a|^{n p} t\right)}\right. \\
& * \mathcal{F}^{\prime}\left(\left(\|a x\|^{p}+\|a y\|^{p}+\|a z\|^{p}+\|w\|^{p}\right) z_{0}, \frac{|2||a|^{10 n}\left(|a|^{10}-|a|^{p}\right)}{\left.\left(1+r_{0}\right)^{p}|a|^{n p} t\right)}\right. \\
\mathcal{P}(D(r x, r y, r z, r w) & \left.-\frac{f\left(a^{n} r x, a^{n} r y, a^{n} r z, a^{n} r w\right)}{a^{10 n}}, t\right) \\
& \leq \mathcal{P}^{\prime}\left(\left(\|x\|^{p}+\|y\|^{p}+\|z\|^{p}+\|w\|^{p}\right) z_{0}, \frac{|2 \| a|^{-9}|a|^{10 n}\left(|a|^{10}-|a|^{p}\right)}{\left(1+r_{0}\right)^{p}|a|^{n p}} t\right) \\
& \diamond \mathcal{P}^{\prime}\left(\left(\|a x\|^{p}+\|y\|^{p}+\|z\|^{p}+\|w\|^{p}\right) z_{0}, \frac{|2||a|^{-7}|a|^{10 n}\left(|a|^{10}-|a|^{p}\right)}{\left(1+r_{0}\right)^{p}|a|^{n p}} t\right) \\
& \diamond \mathcal{P}^{\prime}\left(\left(\|a x\|^{p}+\|a y\|^{p}+\|z\|^{p}+\|w\|^{p}\right) z_{0}, \frac{\left.|2 \|| a\right|^{-4}|a|^{10 n}\left(|a|^{10}-|a|^{p}\right)}{\left(1+r_{0}\right)^{p}|a|^{n p}} t\right) \\
& \diamond \mathcal{P}^{\prime}\left(\left(\|a x\|^{p}+\|a y\|^{p}+\|a z\|^{p}+\|w\|^{p}\right) z_{0}, \frac{|2 \| a|^{10 n}\left(|a|^{10}-|a|^{p}\right)}{\left(1+r_{0}\right)^{p}|a|^{n p}} t\right) .
\end{aligned}\right.
$$

For all $\left|r-r_{0}\right|<1$ and $r \in \mathbb{R}$. Since

$$
\begin{aligned}
& \lim _{n \rightarrow \infty} \frac{|2||a|^{-9}|a|^{10 n}\left(|a|^{10}-|a|^{p}\right)}{\left(1+r_{0}\right)^{p}|a|^{n p}} t=\infty, \\
& \lim _{n \rightarrow \infty} \frac{|2||a|^{-7}|a|^{10 n}\left(|a|^{10}-|a|^{p}\right)}{\left(1+r_{0}\right)^{p}|a|^{n p}} t=\infty, \\
& \lim _{n \rightarrow \infty} \frac{|2||a|^{-4}|a|^{10 n}\left(|a|^{10}-|a|^{p}\right)}{\left(1+r_{0}\right)^{p}|a|^{n p}} t=\infty, \\
& \lim _{n \rightarrow \infty} \frac{|2||a|^{10 n}\left(|a|^{10}-|a|^{p}\right)}{\left(1+r_{0}\right)^{p}|a|^{n p}} t=\infty .
\end{aligned}
$$

Then there exists $n_{0} \in \mathbb{N}$ such that

$$
\left\{\begin{array}{l}
\mathcal{F}\left(D(r x, r y, r z, r w)-\frac{f\left(a^{n_{0}} r x, a^{n_{0}} r y, a^{n_{0}} r z, a^{n_{0}} r w\right)}{a^{10 n_{0}}}, \epsilon\right) \geq \rho \\
\mathcal{P}\left(D(r x, r y, r z, r w)-\frac{f\left(a^{n_{0}} r x, a^{n_{0}} r y, a^{n_{0}} r z, a^{n_{0}} r w\right)}{a^{10 n_{0}}}, \epsilon\right) \leq 1-\rho
\end{array}\right.
$$

For all $\left|r-r_{0}\right|<1$ and $r \in \mathbb{R}$. By the non-Archimedean intuitionistic fuzzy continuity of the mapping $r \rightarrow \frac{f\left(a^{n} 0 r x, a^{n} 0 r y, a^{n} 0 r z, a^{n} 0 r w\right)}{a^{10 n} 0}$, there exists $\delta<1$ such that for each $r$ with $\left|r-r_{0}\right|<\delta$ we have 


$$
\left\{\begin{array}{l}
\mathcal{F}\left(\frac{f\left(a^{n} r x, a^{n} r y, a^{n} r z, a^{n} r w\right)}{a^{10 n}}-\frac{f\left(a^{n_{0}} r_{0} x, a^{n_{0}} r_{0} y, a^{n_{0}} r_{0} z, a^{n_{0}} r_{0} w\right)}{a^{10 n_{0}}}, \epsilon\right) \geq \rho \\
\mathcal{P}\left(\frac{f\left(a^{n} r x, a^{n} r y, a^{n} r z, a^{n} r w\right)}{a^{10 n}}-\frac{f\left(a^{n_{0}} r_{0} x, a^{n_{0}} r_{0} y, a^{n_{0}} r_{0} z, a^{n_{0}} r_{0} w\right)}{a^{10 n_{0}}}, \epsilon\right) \leq 1-\rho .
\end{array}\right.
$$

It follows that

$$
\left\{\begin{array}{l}
\mathcal{F}\left(D(r x, r y, r z, r w)-D\left(r_{0} x, r_{0} y, r_{0} z, r_{0} w\right), \epsilon\right) \geq \rho \\
\mathcal{P}\left(D(r x, r y, r z, r w)-D\left(r_{0} x, r_{0} y, r_{0} z, r_{0} w\right), \epsilon\right) \leq 1-\rho
\end{array}\right.
$$

for each $r$ with $\left|r-r_{0}\right|<\delta$. Hence, the mapping $r \rightarrow D(r x, r y, r z, r w)$ is non-Archimedean intuitionistic fuzzy continuous.

Theorem 4.3. Let $X$ be a non-Archimedean space and let $\left(Z, \mathcal{F}^{\prime}, \mathcal{P}^{\prime}\right)$ be a non-Archimedean IFN space. Let $p<10$ and $z_{0} \in Z$. Let $f$ be a mapping from $X \times X \times X \times X$ to a non-Archimedean intuitionistic fuzzy Banach space $(Y, \mathcal{F}, \mathcal{P})$ such that $f(x, 0, z, w)=0, f(x, y, z, 0)=0$ for all $x, y, z, w \in X$, and satisfying (4.1), (4.2), (4.3), (4.4). Then there exists a unique dectic mapping $D: X \times X \times X \times X \rightarrow Y$ such that

$$
\left\{\begin{aligned}
\mathcal{F}(f(x, y, z, w)-D(x, y, z, w), t) \\
\geq \mathcal{F}^{\prime}\left(\left(\|x\|^{p}+\|y\|^{p}+\|z\|^{p}+\|w\|^{p}\right) z_{0},|2||a|^{-9}\left(|a|^{p}-|a|^{10}\right) t\right) \\
\quad * \mathcal{F}^{\prime}\left(\left(\|x\|^{p}+\|y\|^{p}+\|z\|^{p}+\|w\|^{p}\right) z_{0},|2||a|^{-7}\left(|a|^{p}-|a|^{10}\right) t\right) \\
\quad * \mathcal{F}^{\prime}\left(\left(\|x\|^{p}+\|y\|^{p}+\|z\|^{p}+\|w\|^{p}\right) z_{0},|2||a|^{-4}\left(|a|^{p}-|a|^{10}\right) t\right) \\
\quad * \mathcal{F}^{\prime}\left(\left(\|x\|^{p}+\|y\|^{p}+\|z\|^{p}+\|w\|^{p}\right) z_{0},|2|\left(|a|^{p}-|a|^{10}\right) t\right) \\
\mathcal{P}(f(x, y, z, w)-D(x, y, z, w), t) \\
\leq \mathcal{P}^{\prime}\left(\left(\|x\|^{p}+\|y\|^{p}+\|z\|^{p}+\|w\|^{p}\right) z_{0},|2||a|^{-9}\left(|a|^{p}-|a|^{10}\right) t\right) \\
\diamond \mathcal{P}^{\prime}\left(\left(\|x\|^{p}+\|y\|^{p}+\|z\|^{p}+\|w\|^{p}\right) z_{0},|2||a|^{-7}\left(|a|^{p}-|a|^{10}\right) t\right) \\
\diamond \mathcal{P}^{\prime}\left(\left(\|x\|^{p}+\|y\|^{p}+\|z\|^{p}+\|w\|^{p}\right) z_{0},|2||a|^{-4}\left(|a|^{p}-|a|^{10}\right) t\right) \\
\diamond \mathcal{P}^{\prime}\left(\left(\|x\|^{p}+\|y\|^{p}+\|z\|^{p}+\|w\|^{p}\right) z_{0},|2|\left(|a|^{p}-|a|^{10}\right) t\right)
\end{aligned}\right.
$$

for all $x, y, z, w \in X$ and all $t>0$. Furthermore, if the mapping $g: \mathbb{R} \rightarrow Y$ defined by $g(r):=a^{10 n} f\left(\frac{r x}{a^{n}}\right.$, $\frac{r y}{a^{n}}, \frac{r z}{a^{n}}, \frac{r w}{a^{n}}$, ) is non-Archimedean intuitionistic fuzzy continuous for some $x, y, z, w \in X$ and all $n \in$ $\mathbb{N}$ then the mapping $r \rightarrow D(r x, r y, r z, r w)$ from $\mathbb{R}$ to $Y$ is non-Archimedean intuitionistic fuzzy continuous.

Proof. Define $\varphi_{i}: X \times X \times X \times X \rightarrow Z$ by $\varphi_{i}(x, y, z, w)=\left(\|x\|^{p}+\|y\|^{p}+\|z\|^{p}+\|w\|^{p}+\|v\|^{p}\right) z_{0}$ for all $x, y, z, w, v \in X$ and all $i \in I$. Existence and uniqueness of the dectic mapping $D$ satisfying (4.5) are deduced from Theorem 3.2. Note that, for all $x, y, z, w \in X$, all $t>0, n \in \mathbb{N}$ and all $i \in I$, we have

$\mathcal{F}^{\prime}\left(\varphi_{i}\left(\frac{x}{a}, \frac{y}{a}, \frac{z}{a}, \frac{w}{a}, \frac{v}{a}\right), t\right)=\mathcal{F}^{\prime}\left(\left(\|x\|^{p}+\|y\|^{p}+\|z\|^{p}+\|w\|^{p}+\|v\|^{p}\right) z_{0},|a|^{p} t\right)$

$\mathcal{P}^{\prime}\left(\varphi_{i}\left(\frac{x}{a}, \frac{y}{a}, \frac{z}{a}, \frac{w}{a}, \frac{v}{a}\right), t\right)=\mathcal{P}^{\prime}\left(\left(\|x\|^{p}+\|y\|^{p}+\|z\|^{p}+\|w\|^{p}+\|v\|^{p}\right) z_{0},|a|^{p} t\right)$.

Since $p<10$, we have

$|\alpha|=|a|^{p}>|a|^{p}$. By Theorem 3.1 there exists a unique dectic mapping $D$ which satisfies (4.8). The rest of the proof can be done by the same way as in Theorem 4.1. 


\section{References}

[1] M. A. Abolfathi, A. Ebadian and R. Aghalary, Stability of mixed additive-quadratic Jensen type functional equation in non-Archimedean $\ell$-fuzzy normed spaces, Annali Dell 'Universita 'Di Ferrara. 2013 doi: 10.1007/s11565-013-0182-z, 13 pages, (2013).

[2] T. Aoki, On the stability of the linear transformation in Banach spaces, J. Math. Soc. Japan, 2(1950), 64-66.

[3] K. Atanassov, Intuitionistic fuzzy sets, VII ITKR'S, Session, Sofia, June 1983 (Deposed in Central Science-Technical Library of Bulg. Academy of Science, 1697/84) (in Bulgarian).

[4] A. Ebadian, R. Aghalary and M. A. Abolfathi, Approximation of homomorphisms and derivations of additive functional equation of n-Apollonius type in induced fuzzy Lie $\mathrm{C}^{*}$-algebras, Journal of Advances in Mathematics, 3 (2013), 201-217.

[5] A. Ebadian and S. Zolfaghari, Stability of a mixed additive and cubic functional equation in several variables in non-Archimedean spaces, Annali Dell 'Universita 'Di Ferrara. doi:10.1007/s11565-0120152-x.

[6] M. Eshaghi Gordji, S. Kaboli-Gharetapeh, C. Park and S. Zolfaghari, Stability of an additive-cubicquartic functional equation, Advances in Difference Equations. Article ID 395693, 20 pages, (2009).

[7] M. Eshaghi Gordji, M., Kaboli Gharetapeh, S., Rassias, J. M. and Zolfaghari, S. Solution and stability of a mixed type additive, quadratic and cubic functional equation, Advances in difference equations. Article ID 826130, 17 pages, doi:10.1155/2009/826130, (2009).

[8] M. Eshaghi Gordji, M. S. Moslehian, S. Kaboli and S. Zolfaghari, Stability of a mixed type additive, quadratic, cubic and quartic functional equation, Nonlinear Analysis and variational problems, A. Khan, (ed.) et al., Springer-Verlag, (2009), 65-80.

[9] M. Eshaghi Gordji, S. Zolfaghari, J. M. Rassias and M. B. Savadkouhi, Solution and Stability of a Mixed type Cubic and Quartic functional equation in Quasi-Banach spaces, Abstract and Applied Analysis, Art. ID 417473, (2009), 1-14.

[10] P. Gavruta, A generalization of the Hyers-Ulam-Rassias stability of approximately additive mappings, J. Math. Anal. Appl. 184 (1994),431-436.

[11] K. Hensel, Uber eine neue Begrundung der Theorie der algebraischen Zahlen, Jahresber. Deutsch. Math. Verein. 6(1897), 83-88.

[12] D. H. Hyers, On the stability of the linear functional equation, Proc. Natl. Acad. Sci. 27 (1941), 222224.

[13] S. A. Mohiuddine and Q. M. Danish Lohani, On generalized statistical convergence in intuitionistic fuzzy normed spaces, Chaos Solitions Fractals, 42 (2009), 1731-1737. 
[14] S. A. Mohiuddine and H. Sevli, Stability of Pexiderized quadratic functional equation in intuitionistic fuzzy normed space, Journal of Computational and Applied Mathematics, 235 ( 8) (2011) 2137- 2146.

[15] S. A. Mohiuddine, M. Cancan and H. Sevli, Intuitionistic fuzzy stability of a Jensen functional equation via fixed point technique, Mathematical and Computer Modelling,54 (9-10) (2011), 2403-2409.

[16] S. A. Mohiuddine, Stability of Jensen functional equation in intuitionistic fuzzy normed space, Chaos, Solitons and Fractals, 42( 5)(2009), 2989-2996.

[17] M. Mursaleen and S. A. Mohiuddine On stability of a cubic functional equation in intuitionistic fuzzy normed spaces, Chaos, Solitons and Fractals, 42 (5) (2009), 2997-2005.

[18] S. A. Mohiuddine and M. A. Alghamdi, Stability of functional equation obtained through fixed point alternative in intuitionistic fuzzy normed spaces, Advances in Difference Equations, 2012, article 141, (2012).

[19] M. Mursaleen and S. A. Mohiuddine, Nonlinear operators between intuitionistic fuzzy normed spaces and Fr_echet derivative, Chaos, Solitons and Fractals. 42(2009), 1010-1015.

[20] M. Mursaleen and S. A. Mohiuddine, On lacunary statistical convergence with respect to the intuitionistic fuzzy normed spaces, J. Comput. Appl. Math. 233(2009), 141-149.

[21] Th. M. Rassias, On the stability of the linear mapping in Banach spaces, Proc. Amer. Math. Soc. 72(1978), 297-300.

[22] R. Saadati, and J. H. Park, On the intuitionistic fuzzy topological spaces, Chaos, Solitons and Fractals, 27( 2)(2006), 331-344.

[23] Z. Wang and T. M. Rassias, Intuitionistic fuzzy stability of functional equations associated with inner product spaces, Abstract and Applied Analysis, vol. 2011, Article ID 456182, 19 pages, (2011).

[24] T. Z. Xu, J. M. Rassias and W. X. Xu, Intuitionistic fuzzy stability of a general mixed additive-cubic equation, Journal of Mathematical Physics, 51(6), Article ID 063519, (2010).

[25] S. M. Ulam, A Collection of the mathematical problems. Interscience, New York, 1960. 